\title{
Optimizing Sparse Matrix-Vector Multiplications on An ARMv8-based Many-Core Architecture
}

\author{
Donglin Chen • Jianbin Fang • Shizhao \\ Chen · Chuanfu Xu • Zheng Wang
}

Received: date / Accepted: date

\begin{abstract}
Sparse matrix-vector multiplications (SpMV) are common in scientific and HPC applications but are hard to be optimized. While the ARMv8based processor IP is emerging as an alternative to the traditional x64 HPC processor design, there is little study on SpMV performance on such new many-cores. To design efficient HPC software and hardware, we need to understand how well SpMV performs. This work develops a quantitative approach to characterize SpMV performance on a recent ARMv8-based many-core architecture, Phytium FT-2000 Plus (FTP). We perform extensive experiments involved over 9,500 distinct profiling runs on 956 sparse datasets and five mainstream sparse matrix storage formats, and compare FTP against the Intel Knights Landing many-core. We experimentally show that picking the optimal sparse matrix storage format and parameters is non-trivial as the correct decision requires expert knowledge of the input matrix and the hardware. We address the problem by proposing a machine learning based model that predicts the best storage format and parameters using input matrix features. The model automatically specializes to the many-core architectures we considered. The experimental results show that our approach achieves on average $93 \%$ of the best-available performance without incurring runtime profiling overhead.
\end{abstract}

Keywords SpMV Sparse matrix format · Many-Core $\cdot$ Performance tuning

D. Chen, J. Fang, S. Chen, C. Xu are with College of Computer Science, National University of Defense Technology, Changsha 410073, China.

E-mail: \{chendonglin14, j.fang, chenshizhao12, xuchuanfu\}@nudt.edu.cn .

Z. Wang is with School of Computing and Communications, Lancaster University, Lancaster LA1 4WA, United Kingdom.

E-mail: z.wang@lancaster.ac.uk 


\section{Introduction}

The sparse matrix-vector multiplication $(\mathrm{SpMV})^{1}$ is one of the most common operations in scientific and high-performance-computing (HPC) applications [35]. While SpMV is often responsible for the application performance bottleneck, it is notoriously difficult to be optimized. This is due to a number of inherent issues arising from the computation kernel, the matrix storage format, the sparsity pattern of the input matrix, and the complexity of parallel hardware [3, 4, 5, 22, 23, 25, 49].

Numerous sparse matrix storage formats have been proposed $[2,16,17,23$, $28,47]$, all aiming to reduce the memory footprint by only storing a fraction of the elements of the target matrix. While there is an extensive body of work on optimizing SpMV on SMP and multi-core architectures [23, 25], there is little work on investigating SpMV performance on the ARMv8-based many-core architectures. Given that the ARMv8-based processor IP is emerging as an alternative for HPC processor architecture [18, 36, 50], it is crucial to understand how well different sparse matrix storage formats perform on such architectures and what affects the resulting performance. Understanding this can not only help software developers to write better code for the next-generation HPC systems, but also provide useful insights for hardware architects to design more efficient hardware for this important application domain.

In this paper, we investigate the SpMV performance on the latest ARMv8based Phytium FT-2000 Plus (FTP) $[1,50]$. This architecture integrates over 60 processor cores to offer a powerful computation capability, making it attractive for the next-generation HPC systems. We conduct a large-scale evaluation involved over 9,500 profiling runs performed on 956 representative sparse datasets and consider five widely-used sparse matrix representations: CSR [47], CSR5 [23], ELL [16], SELL [17, 28], and HYB [2]. We also compare SpMV performance on FTP against the Intel Knights Landing (KNL) multi-core that has been deployed in many HPC systems. This comparison provides insights on whether an ARMv8-based many-core requires a different optimization strategy for SpMV computation.

We demonstrate that although there is significant gain for choosing the right sparse matrix storage format and parameters, mistakes can seriously hurt the performance. We then investigate what cause the performance disparity. Our data show that picking the optimal storage format and parameters requires expert knowledge of the underlying hardware and the input matrix. To help developers to choose the right storage format, we employ machine learning to develop a predictive model. Our model is trained offline using a set of training examples. The inputs to the model are static features extracted from the input matrix. The trained model is then used at runtime to choose the optimal storage format for any unseen sparse matrix. Experimental results show that our approach is highly effective in choosing the sparse matrix stor-

1 A SpMV operation $-\mathbf{y}=\mathbf{A} \mathbf{x}-$ multiplies a sparse matrix $\mathbf{A}$ of size $m \times n$ by a dense vector $\mathbf{x}$ of size $n$, and then produces a dense vector $\mathbf{y}$ of size $m$. 
age format, delivering on average over $90 \%$ of the best-available performance on FTP and KNL.

In summary this paper makes the following contributions:

- We provide the first extensive characterization of SpMV performance on FTP, an emerging ARMv8-based many-core architecture for HPC;

- We reveal how the storage format parameters and hardware architecture differences affect the SpMV performance on FTP and KNL;

- We develop a machine learning technique to predict the best sparse matrix storage format, which is portable across many-core architectures.

\section{Background and Experimental Setup}

In this section, we describe the sparse matrix storage formats considered in this work and our experimental setup.

\subsection{Sparse Matrix Storage Formats}

We consider five mainstream sparse matrix storage formats, described as follows.

CSR. The compressed sparse row (CSR) format explicitly stores column indices and nonzeros in arrays indices and data, respectively. It uses a vector ptr, which points to row starts in indices and data, to query matrix values. The length of ptr is $n_{-} r o w+1$, where the last item is the total number of the nonzero elements of the matrix.

CSR5. The CSR5 format aims to obtain a good load balance for matrix value queries [23]. It achieves this by partitioning all nonzero elements into multiple 2-dimensional tiles of the same size.

ELL. For an $M \times N$ matrix with a maximum number of $K$ nonzero elements per row, The ELLPACK-Itpack (ELL) format stores the sparse matrix in a dense $M \times K$ array. If there are fewer than $K$ elements in a row, the row is padded with zeros. ELL uses an integer companion array, indices, to store the column indices of the each nonzero element. This scheme may be inefficient if many rows of the target matrix have fewer than $K$ elements.

SELL. Sliced ELL (SELL) is an extension to the ELL format by partitioning the input matrix into strips of $C$ adjacent rows [28]. Each strip is stored in the ELL format but the number of nonzero elements of each strip may be different. Because the number of stored elements in each row is no longer determined by the maximum of nonzero elements of a row but by the "longest row" in this strip of rows, some of the slices may require less storage space compared to ELL. SELL-C- $\sigma$ improves the vanilla SELL by adding row sorting such that rows with similar number of nonzero elements are grouped in one block [17]. To trade-off the cost of sorting against the acceleration of the SpMV, rows 


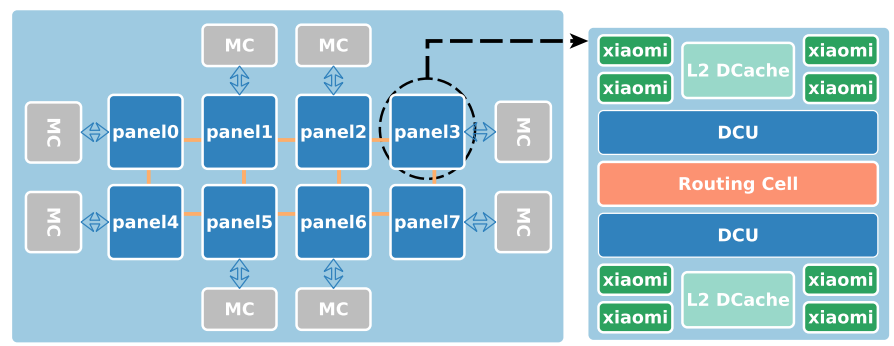

Fig. 1 A high-level overview of the FTP architecture.

are not sorted globally but within $\sigma$ consecutive rows. In this work, we use SELL-C- $\sigma$ and refers it as SELL to aid readability thereafter.

HYB. The HYB format is a combination of ELL and the simple COO format which explicitly stores indices of the row, column, and values of nonzero elements [2]. For each matrix row, HYB stores $K$ nonzero elements using the ELL format and the remaining elements in COO.

\subsection{Evaluation Setup}

Hardware Platforms. As depicted in Figure 1, FTP integrates 64 ARMv8 based Xiaomi cores. It offers a peak performance of 512 Gflops for doubleprecision operations, with a maximum power consumption of 100 Watts. The cores can run up to $2.4 \mathrm{GHz}$, and are groups into eight panels with eight cores per panel. Each core has a private $32 \mathrm{~KB}$ L1 data cache, and a $2 \mathrm{MB}$ L2 cache shared among four cores. The panels are connected through two directory control units (DCU) [1]. In addition to FTP, we also evaluate the SpMV performance on the Intel KNL many-core. This allows us to directly compare an ARMv8-based architecture against the popular Intel-based manycore design. A KNL chip integrates 72 cores where each core has four threads running at $1.3 \mathrm{GHz}$.

Systems Software. We run a customized Linux OS with Linux Kernel v4.4 and v3.10 on FTP and KNL respectively. For compilation, we use gcc v6.4.0 on FTP and Intel icc v17.0.4 on KNL with the "-O3" compiler option. We use the OpenMP threading model, using 64 threads on FTP and 72 threads on KNL.

Datasets. We use 956 square matrices (with a total size of $90 \mathrm{~GB}$ ) from the SuiteSparse matrix collection [8]. The number of nonzero elements of the matrices ranges from $100 \mathrm{~K}$ to $200 \mathrm{M}$ [21]. The dataset includes both regular and irregular matrices, covering domains from scientific computing to social networks [24]. 


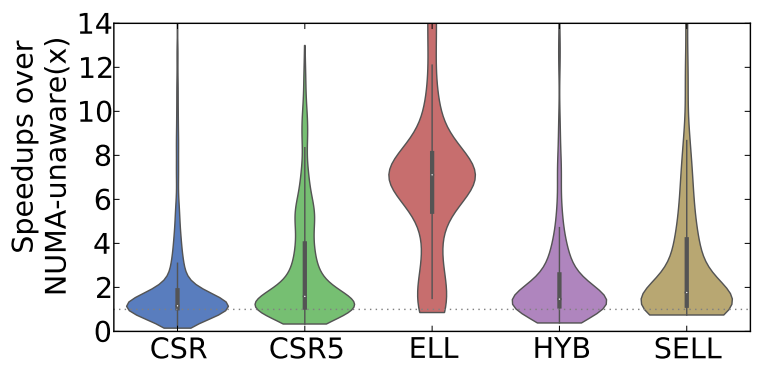

Fig. 2 The violin diagram shows the speedup distribution of NUMA-aware memory allocation on FTP. The thick black line shows where $50 \%$ of the data locates.

\section{SpMV Performance Analysis}

\subsection{Roadmap}

Recall that our goal is to understand how different sparse matrix storage formats perform on FTP. In addition to the sparse matrix storage format, memory allocation and code optimization can also affect the SpMV performance. To isolate the problem, we need to find out the optimal memory allocation and code optimization scheme. For memory allocation, we investigate NonUniform Memory Access (NUMA) bindings. For code optimization, we look at vectorization because it is an important optimization opportunity for matrix multiplications. We then study the impact of the sparse matrix storage format by using the best-found strategy of NUMA memory allocation and code vectorization.

\subsection{The Impact of NUMA Bindings}

FTP exposes eight NUMA nodes where a group of eight cores are directly connected to a local memory module. Indirect access to remote memory modules is possible but $1.5 \mathrm{x}$ slower than accessing the local module. Here we use the Linux NUMA utility, numactl, to allocate the required data buffers from the local memory module for an OpenMP thread that performs SpMV computation [14].

As can be seen from Figure 2, NUMA-aware memory allocation significantly outperforms the non-NUMA-aware counterpart, giving an average speedup ranging from $1.5 \mathrm{x}$ to $6 \mathrm{x}$ across five storage formats. As such, we enable static NUMA bindings on FTP. We also observe that the ELL format consumes the largest memory buffers among the five storage formats, and thus we can achieve the maximum speedup with manual NUMA bindings. 


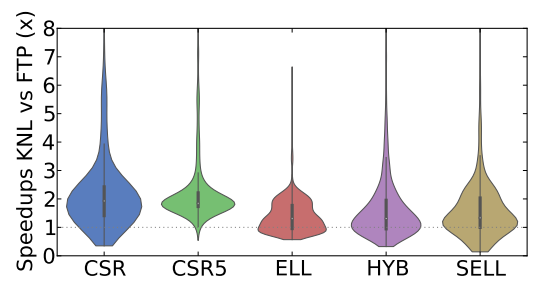

(a) KNL over FTP

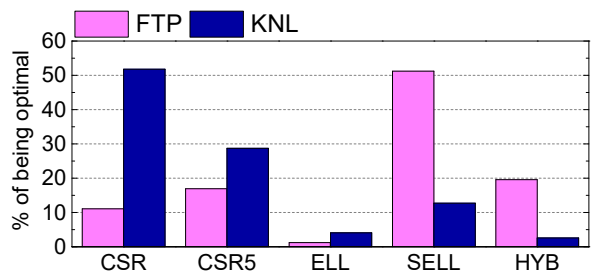

(b) Optimal storage format distribution

Fig. 3 Sub-figure (a) shows the speedups of KNL over FTP and sub-figure (b) suggests the optimal storage format changes from one architecture to the other.

\subsection{The Impact of Code Vectorization}

To investigate the impact of vectorization, we manually vectorize the computational kernel using the CSR5 and the SELL storage formats. Before testing our code on FTP, we verify it on KNL which also has a SIMD vectorization unit. We obtain a speedup of $1.6 \mathrm{x}$ and $1.5 \mathrm{x}$ for CSR5 and SELL respectively over the non-vectorized code, confirming the effectiveness of our manual implementation. However, we observe no speedup and sometimes slowdown for running the vectorized code on FTP. We believe this is because unlike KNL, FTP does not support the gather operation which is essential for accessing elements from different locations of a vector. Our findings suggest that future ARMv8-based many-core designs perhaps should support the gather operation to achieve good vectorization performance. For the remaining experiments in this work, we use the manually vectorized code on KNL and the non-vectorized code on FTP.

\subsection{The Impact of Hardware Architecture Differences}

Figure 3(a) compares the performance by running the same kernel on KNL over FTP. KNL outperforms FTP by delivering, on average, at least $1.3 \mathrm{x}$ speedup (up to $2.1 \mathrm{x}$ ) across the five storage formats. The performance advantage of KNL primarily comes from its Multi-Channel DRAM (MCDRAM) which provides more than $6 \mathrm{x}$ bandwidth over the traditional DDR memory. Thus, by providing a higher bandwidth, MCDRAM significantly reduces the memory access time and brings great benefits to sparse kernels once the data is loaded into it [19]. The performance benefit of KNL also comes from the better support of code vectorization as mentioned in Section 3.3. On the other hand, we observe that on some matrices, especially when the matrix size is small, FTP delivers better performance over KNL. This is largely due to the larger L2 data cache on FTP and a more efficient coherence protocol. Overall, our results suggest that a fast memory hierarchy is essential for obtaining good SpMV performance. 
Table 1 The average slowdown (x) over the optimal when using a fixed storage format.

\begin{tabular}{llllll}
\hline & CSR & CSR5 & ELL & SELL & HYB \\
\hline FTP & $1.5 \mathrm{x}$ & $1.7 \mathrm{x}$ & $6.6 \mathrm{x}$ & $1.3 \mathrm{x}$ & $1.2 \mathrm{x}$ \\
KNL & $1.3 \mathrm{x}$ & $1.4 \mathrm{x}$ & $8.7 \mathrm{x}$ & $1.5 \mathrm{x}$ & $1.6 \mathrm{x}$ \\
\hline
\end{tabular}

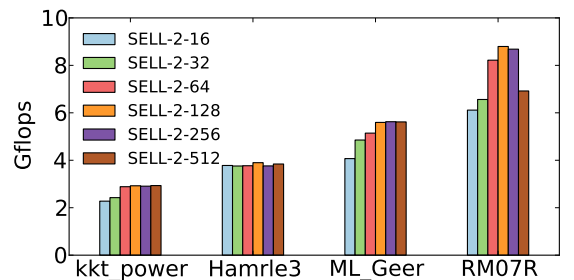

(a) Perf. when $\sigma$ increases and $C=2$

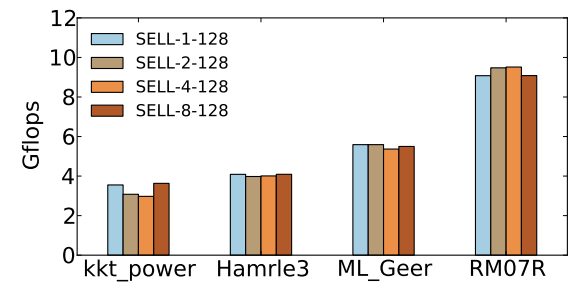

(b) Perf. when $C$ increases and $\sigma=128$

Fig. 4 The impact of $\sigma$ and $\mathrm{C}$ on SpMV performance.

Figure 3(b) shows the optimal storage format distribution changes from one architecture to the other. For example, although CSR is optimal for more than half of the matrices on KNL, it should only be used for $10 \%$ of the matrices on FTP. This diagram suggests that the choice of the storage format depends on the underlying hardware. Table 1 gives the average slowdowns when using a fixed format across all test cases over the optimal one. The slowdown has a negative correlation with how often a given format being optimal. Using a fixed format can miss significant optimization opportunities with up to $6.6 \mathrm{x}$ and $8.7 \mathrm{x}$ slowdowns on FTP and KNL respectively. This experiment shows that there is no "one-fits-for-all" storage format across matrices and architectures. As such we need to have an adaptive scheme to help developers to choose the optimal sparse matrix format. In Section 4, we describe how to develop such an approach using machine learning.

\subsection{The Impact of Storage Format Parameters}

We now consider the impact of choosing storage format parameters. Among the five storage formats considered in this work, SELL has two tuning parameters, $C$ and $\sigma$, and HYB has one tuning parameter, $K$. In this experiment, we investigate the impact of these tuning parameters on FTP.

\subsubsection{SELL}

We choose four matrices, RM07R, kkt power, Hamrle3, and ML Geer, to evaluate how different values of $C$ and $\sigma$ affect the performance of SELL. These four matrices are chosen because they represent distinct matrix characteristics. 


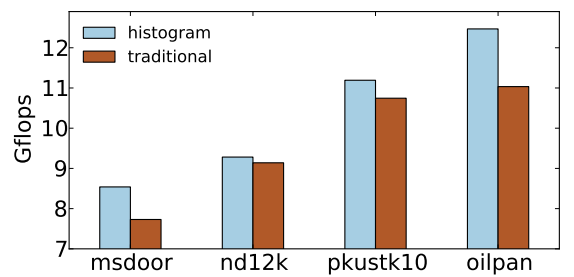

(a) Performance with different strategies

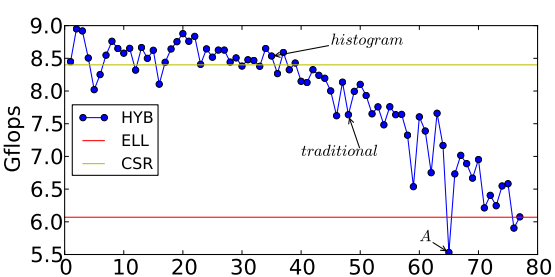

(b) Performance when $\mathrm{K}$ increases

Fig. 5 How the change of $K$ of HYB affects the SpMV performance.

Figure 4(a) shows the resulting performance as $\sigma$ increases when we fix $C$ to 2 (which matches the double-precision register width of FTP). We observe improved performance for all matrices when using a larger $\sigma$, which in turns leads to less padding operations (see Section 2.1), but the performance improvement reaches a plateau when $\sigma$ is set to 128. This is because a larger $\sigma$ also means a bigger sorting scope, which is more likely to increase the load imbalance.

Figure 4(b) shows how the change of $C$ affects the performance. In this experiment, we fix $\sigma$ to the overall optimal value of 128 . Here, we observe little change in performance with different $C$ values. This is because while a larger $C$ enables more aggressive loop unrolling (which can improve performance), it also incurs more padding operations which can eclipse the benefit of loop unrolling.

\subsection{2 $H Y B$}

Recall that HYB stores $K$ nonzero elements in ELL and the rest in COO. Thus, the choice of $K$ can have an impact of the SpMV performance. In this experiment, we compare two algorithms for choosing $K$ : an average based algorithm [6] and a "histogram" based scheme [2]. This evaluation is performed on four matrices listed in Figure 5(a) to keep the experiments manageable. Note that these matrices are different from those used to study SELL, because these are the matrices where HYB is the optimal choice.

As can be seen from Figure 5(a), the "histogram" based algorithm delivers, on average, $10 \%$ performance improvement over its counterpart. Figure 5(b) shows how the performance on msdoor changes when $K$ is increased from 1 to 80 . HYB could be a good storage format for the considered matrices, but this requires ones to choose the correct $K$ value. A wrong $K$ value can lead to significantly worse performance, e.g., the point marked with label $A$ in Figure 5(b). This example shows how important it is to choose the right parameter setting. 


\section{Predictive Modeling for Storage Format Selection}

We develop an automatic machine learning approach to automatically choose the correct sparse matrix storage format. Our approach takes a new, unseen sparse matrix and is able to predict the optimal or near optimal sparse matrix representation for a given architecture. To demonstrate the portability of our approach, we train and evaluate a predictive model on FTP and KNL.

Our model for predicting the best sparse matrix storage format is a random forest model that consists of multiple decision trees [13]. We have evaluated other alternative techniques, including regression, Naive Bayes and K-Nearest neighbour (see also Section 5.2). We chose the decision tree model because it gives the best performance and can be easily interpreted compared to other black-box models.

Building and using such a model follows the 3-step process for supervised machine learning: (i) generate training data (ii) train a predictive model (iii) use the predictor, described as follows. Our predictive model is built upon the Python scikit-learn package [31].

\subsection{Training the Predictor}

To train a predictor we first need to find the best sparse matrix storage format for each of our training examples, and extract features. We then use this set of data and classification labels to train our predictor model.

Generating Training Data. We use the standard five-fold-cross validation for training. Specifically, we select, from the SuiteSparse matrix collection, $20 \%$ samples for testing and then use $80 \%$ samples (i.e., 756 matrices) for training. We execute SpMV using each of the targeting sparse matrix storage formats. We run each training setting several times until the gap of the upper and lower confidence bounds is smaller than $5 \%$ under a $95 \%$ confidence interval setting. We then record the best-performing storage format for each training sample on our target hardware platform. Finally, we extract the values of our selected set of features from each matrix.

Building The Model. The optimal matrix storage labels, along with their corresponding feature set, are passed to our supervised learning algorithm. The learning algorithm tries to find a correlation between the feature values and optimal representation labels. The output of our learning algorithm is a version of our random forests model.

Total Training Time. The total training time of our model is comprised of two parts: gathering the training data, and then building the model. Gathering the training data consumes most of the total training time, in this paper it took around 3 days for the FTP and KNL platforms. In comparison actually building the model took a negligible amount of time, less than $10 \mathrm{~ms}$. Since training is performed off-line and only need to be carried out once for a given architecture, this is a one-off cost. 
Table 2 The features used in our model.

\begin{tabular}{llll}
\hline Features & Description & Features & Description \\
\hline n_rows & number of rows & n_cols & number of columns \\
nnz_frac & \% nonzeros & nnz_min & minimum \#nonzeros per row \\
nnz_max & maximum \# nonzeros per row & nnz_avg & average \#nonzeros per row \\
nnz_std & standard derivation \# nonzeros per row & nnz_var & variance \# nonzeros per row \\
\hline
\end{tabular}

\subsection{Features}

Our predictive model is based exclusively on static features of the target matrix and no dynamic profiling is required. Since our goal is to develop a portable, architecture-independent approach, we do not use any hardware-specific features.

We considered a total of seven candidate raw features (Table 2) in this work. Some features were chosen from our intuition based on factors that can affect SpMV performance e.g. $n n z_{-} f r a c$ and $n n z_{-} v a r$, other features were chosen based on previous work [35]. Before passing the feature values to the predictive model, we also scale each scalar value of the feature vector to a common range (between 0 and 1) in order to prevent the range of any single feature being a factor in its importance. We record the minimum and maximum values of each feature in the training dataset, and use these to scale the corresponding features for an unseen input during deployment.

\subsection{Runtime Deployment}

The trained model is encapsulated in a runtime library. We provide an API to extract matrix features and a tool to perform matrix format transformation. For a given matrix, our tool automatically translates it to the five targeted storage formats of parameter settings at compile time. Since the transformation is performed offline, it does not incur runtime overhead. During runtime, the off-line trained model predicts the optimal storage format and parameters to use, and the library automatically selects the offline generated format to run on the target architecture.

\section{Predictive Modeling Evaluation}

\subsection{Overall Performance}

As described in Section 4.1, we use cross-validation to train and test our predictive model to make sure the model is evaluated on new, unseen inputs. We repeat the cross-validation process multiple times to ensure all matrices in our dataset are tested at least once.

Figure 6 shows that our predictor achieves, on average, $93 \%$ and $95 \%$ of the best available SpMV performance (found through exhaustive search) on FTP 


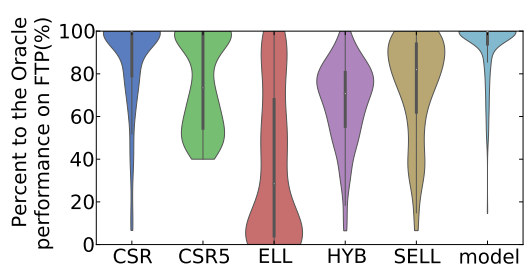

(a) FTP

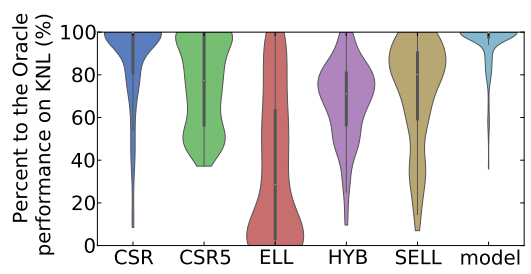

(b) KNL

Fig. 6 The predicted performance of SpMV on FTP and KNL. We show the achieved performance with respect to the best available performance across sparse formats.

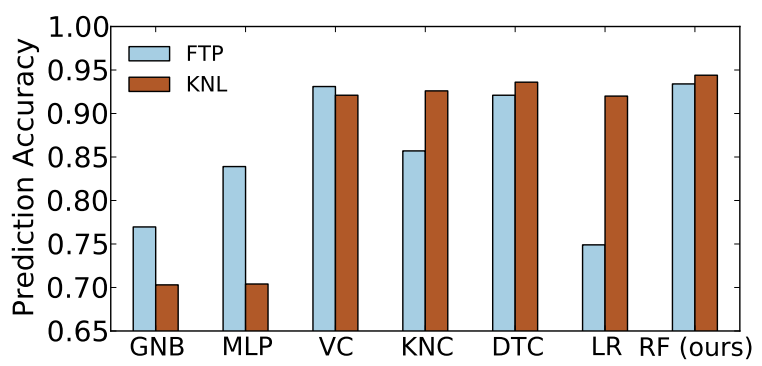

Fig. 7 Compare to alternative classifiers.

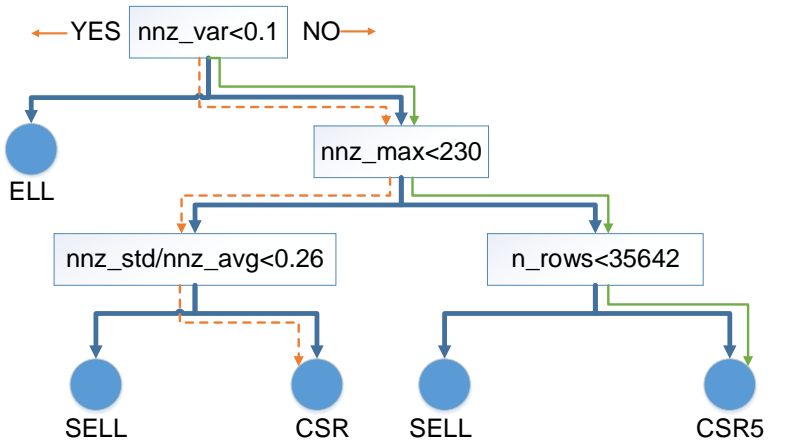

Fig. 8 How two unseen matrices follow the different paths of a learned tree.

and KNL respectively. We also note that our predictor outperforms a strategy that uses only the single overall-best format on each platform, i.e., SELL or HYB on FTP and CSR on KNL (see Table 1). This experiment shows that our predictor is highly effective in choosing the right sparse matrix representation.

\subsection{Alternative Modeling Techniques}

Figure 7 shows resulting performance with respect to the best available performance when using different techniques to construct the predictive model. 
Table 3 Feature values of matrix c-71 and skirt.

\begin{tabular}{llrlrlrlr}
\hline Matrix & Feature & value & Feature & Value & Features & Value & Feature & Value \\
\hline \hline c-71 & n_rows & 76638 & n_cols & 76638 & nnz_frac & 0.000146 & nnz_min & 2 \\
\multirow{2}{*}{ skirt } & nnz_max & 6720 & nnz_avg & 11.2 & nnz_std & 29.2 & nnz_var & 854.7 \\
& n_rows & 12598 & n_cols & 12598 & nnz_frac & 0.00123 & nnz_min & 1 \\
& nnz_max & 33 & nnz_avg & 15.6 & nnz_std & 6.3 & nnz_var & 39.8 \\
\hline
\end{tabular}

In addition to our random forests based model (RF), we also consider Gaussian naïve bayes (GNB), multilayer perception (MLP), soft voting/majority rule Classification (VC), k-Nearest Neighbor (KNC, $\mathrm{k}=1$ ), logistic regression (LR), decision tree classification (DCT). Thanks to the high-quality features, all classifiers are highly accurate in choosing sparse matrix representation. We choose RF because its accuracy is comparable to alternative techniques.

\subsection{Analysis of the Predictive Model}

One of our motivations for using a decision-tree-based model (i.e., random forests) is that this modeling technique is interpretable. This means that we can gain insights of why a certain storage format is chosen.

Figure 8 shows one of the decision trees in our random-forest model on FTP. The learning algorithm automatically places the most relevant features at the root level and determines the architecture-dependent threshold for each node. All this is done automatically without the need of expert intervention.

Table 3 lists the feature values extracted from two distinct matrices, c-71 and skirt. To choose a storage format, we follow the decision tree depicted in Figure 8. At the root of the tree, we look at the value for the nnz_var. This feature uses the variation (i.e., dispersion) for the number of nonezero elements among rows to measure the matrix regularity. The values are far above the threshold, suggesting that the nonzero elements are not evenly distributed in both matrices. We thus go to the right subtree and reach the second level of the tree. This node looks at nnz_max. The feature value of $c-71$ is larger than the threshold and therefore the right branch is taken, but for skirt we choose the left branch. The metric of nnz_max counts the largest number of nonzero elements within a row. A large value in the feature suggests that the longest row is likely to cause load imbalance. In such a case, storage formats like CSR5 and SELL may be a good fit because they are designed to avoid load imbalance. At the second-last level of the tree, we look at nnz_rows and nnz_std / nnz_avg respectively for c-71 and skirt. Based on the feature value of the matrices, we choose CSR5 and CSR respectively for c-71 and skirt. The chosen formats are indeed the optimal storage formats for the two matrices. 


\section{Related Work}

A large body of work has been conducted in the past to study SpMV performance on parallel systems $[27,32,47]$. However, our work is the first comprehensive study for SpMV performance on an ARMv8-based many-core. Our work fills the gap by providing an in-depth performance analysis on two emerging many-core architectures (KNL and FTP). The insights will be useful for designing more efficient parallel HPC software and hardware in the future.

Efforts have been made in designing new storage formats for various parallel processor architectures including SIMD CPUs and SIMT GPUs [2, 15, 23, 25, $26,47,48]$. However, how well these existing sparse matrix formats perform on ARM-based many-cores remains an open problem. Our work attempts to answer this question by providing comprehensive analysis and new insights.

It is shown that there is no universally optimal sparse matrix storage format [52]. Thus, it is important to choose the right format according to the right input matrix features to achieve good SpMV performance. Prior work has developed methods to choose a sparse matrix storage format $[20,35]$, but no work has targeted an ARM-based many-core architecture. Recently, Zhao et al. employ deep learning to automatically extract important features from the input matrices to help to build a predictive model [52]. Their approach of feature extraction is thus orthogonal to our machine learning based approach. Our prior work shows that selecting the right SpMV format on ARM-based many-cores is non-trivial [6]. This work builds upon our past work to show how machine learning can be employed to obtain a deep insights on how to optimize SpMV on such architectures.

Machine learning has been employed for various optimization tasks [40], including code optimization [7, 12, 29, 30, 37, 39, 41, 42, 43, 44, 45, 46, 51], task scheduling $[9,10,11,33,34]$, model selection [38], etc.

Although SpMV optimization has been extensively studied, it remains unclear how the widely-used sparse matrix representations perform on the emerging many-core architectures. Our work aims to bridge this gap by providing an in-depth analysis on two emerging many-core architectures (KNL and FTP), with a large number of sparse matrices and five well-recognized storage formats. Our work is the first attempt in applying machine learning techniques to optimize SpMV on FTP.

\section{Conclusion}

This paper has presented a large-scale study of SpMV performance on an emerging ARMv8-based many-core architecture, Phytium FT-2000Plus (FTP). We show how the memory allocation scheme, code vectorization and the sparse storage format and its parameters affect the SpMV performance. We compare the results on FTP against the ones obtained from another representative HPC processor, the Intel Knights Landing. We reveal how the architectural differences affect the optimization strategies, providing useful insights into the 
possible improvements for the future ARM-based many-core design. Because there is no "one-fits-for-all" sparse matrix storage format, we develop a machine learning based model to help developers to choose the correct format. Our model is first trained offline and the learnt model can be used for any unseen input matrices. Experimental results show that our model is effective and portable across architectures, delivering over $90 \%$ of the best-available performance.

Acknowledgements This work was partially funded by the National Key R\&D Program of China under Grant No. 2017YFB0202003, the National Natural Science Foundation of China under grant agreements 61602501, 11502296, 61772542, 61561146395 and 61872294 ; the Open Research Program of China State Key Laboratory of Aerodynamics under grant agreement SKLA20160104; the UK Engineering and Physical Sciences Research Council under grants EP/M01567X/1 (SANDeRs) and EP/M015793/1 (DIVIDEND); and the Royal Society International Collaboration Grant (IE161012). For any correspondence, please contact Jianbin Fang (Email: j.fang@nudt.edu.cn)

\section{References}

1. (2017) FT-2000. Phytium Technology Co. Ltd., http://www.phytium. com.cn/Product/detail? language=1\&product_id=7

2. Bell N, Garland M (2009) Implementing sparse matrix-vector multiplication on throughput-oriented processors. In: SC

3. Che Y, Xu C, Fang J, Wang Y, Wang Z (2015) Realistic performance characterization of CFD applications on intel many integrated core architecture. Comput J

4. Chen J, Fang J, Liu W, Tang T, Chen X, Yang C (2017) Efficient and portable ALS matrix factorization for recommender systems. In: IPDPS

5. Chen J, Fang J, Liu W, Tang T, Yang C (2018) clmf: A fine-grained and portable alternating least squares algorithm for parallel matrix factorization. FGCS

6. Chen S, Fang J, Chen D, Xu C, Wang Z (2018) Adaptive optimization of sparse matrix-vector multiplication on emerging many-core architectures. In: HPCC '18

7. Cummins C, et al. (2017) End-to-end deep learning of optimization heuristics. In: PACT ' 17

8. Davis TA, Hu Y (2011) The university of florida sparse matrix collection. ACM Trans Math Softw

9. Emani MK, et al. (2013) Smart, adaptive mapping of parallelism in the presence of external workload. In: CGO '13

10. Grewe D, et al. (2011) A workload-aware mapping approach for dataparallel programs. In: HiPEAC '11

11. Grewe D, et al. (2013) Opencl task partitioning in the presence of gpu contention. In: LCPC '13

12. Grewe D, et al. (2013) Portable mapping of data parallel programs to opencl for heterogeneous systems. In: CGO '13 
13. Ho TK (1995) Random decision forests. In: ICDAR, pp 278-282

14. Hollowell C, et al. (2015) The effect of numa tunings on cpu performance. Journal of Physics: Conference Series

15. Im E, Yelick KA, Vuduc RW (2004) Sparsity: Optimization framework for sparse matrix kernels. IJHPCA

16. Kincaid D, et al. (1989) Itpackv 2d user's guide. Tech. rep., Center for Numerical Analysis, Texas Univ., Austin, TX (USA)

17. Kreutzer M, Hager G, Wellein G, Fehske H, Bishop AR (2014) A unified sparse matrix data format for efficient general sparse matrix-vector multiplication on modern processors with wide SIMD units. SIAM J Scientific Computing

18. Laurenzano MA, Tiwari A, Cauble-Chantrenne A, Jundt A, Jr WAW, Campbell RL, Carrington L (2016) Characterization and bottleneck analysis of a 64-bit armv8 platform. In: ISPASS

19. Li A, Liu W, Kristensen MRB, Vinter B, Wang H, Hou K, Marquez A, Song SL (2017) Exploring and analyzing the real impact of modern onpackage memory on HPC scientific kernels. In: SC

20. Li J, Tan G, Chen M, Sun N (2013) SMAT: an input adaptive auto-tuner for sparse matrix-vector multiplication. In: PLDI

21. Liu J, He X, Liu W, Tan G (2018) Register-based implementation of the sparse general matrix-matrix multiplication on gpus. In: PPoPP

22. Liu W (2015) Parallel and scalable sparse basic linear algebra subprograms. PhD thesis, University of Copenhagen

23. Liu W, Vinter B (2015) CSR5: an efficient storage format for crossplatform sparse matrix-vector multiplication. In: ICS

24. Liu W, Vinter B (2015) Speculative segmented sum for sparse matrixvector multiplication on heterogeneous processors. Parallel Computing 49:179-193

25. Liu X, Smelyanskiy M, Chow E, Dubey P (2013) Efficient sparse matrixvector multiplication on $\mathrm{x} 86$-based many-core processors. In: ICS

26. Maggioni M, Berger-Wolf TY (2013) An architecture-aware technique for optimizing sparse matrix-vector multiplication on gpus. In: ICCS

27. Mellor-Crummey JM, Garvin J (2004) Optimizing sparse matrix - vector product computations using unroll and jam. IJHPCA

28. Monakov A, Lokhmotov A, Avetisyan A (2010) Automatically tuning sparse matrix-vector multiplication for GPU architectures. In: HIPEAC

29. Ogilvie WF, et al. (2014) Fast automatic heuristic construction using active learning. In: LCPC '14

30. Ogilvie WF, et al. (2017) Minimizing the cost of iterative compilation with active learning. In: CGO '17

31. Pedregosa F, et al. (2011) Scikit-learn: Machine learning in Python. Journal of Machine Learning Research

32. Pinar A, Heath MT (1999) Improving performance of sparse matrix-vector multiplication. In: SC

33. Ren J, et al. (2017) Optimise web browsing on heterogeneous mobile platforms: a machine learning based approach. In: INFOCOM '17 
34. Ren J, et al. (2018) Adaptive web browsing on mobile heterogeneous multicores. IEEE Computer Architecture Letters

35. Sedaghati N, Mu T, Pouchet L, Parthasarathy S, Sadayappan P (2015) Automatic selection of sparse matrix representation on gpus. In: ICS

36. Stephens N (2016) Armv8-a next-generation vector architecture for HPC. In: 2016 IEEE Hot Chips 28 Symposium (HCS), pp 1-31

37. Taylor B, et al. (2017) Adaptive optimization for opencl programs on embedded heterogeneous systems. In: LCTES '17

38. Taylor B, et al. (2018) Adaptive deep learning model selection on embedded systems. In: LCTES '18

39. Tournavitis G, et al. (2009) Towards a holistic approach to autoparallelization: Integrating profile-driven parallelism detection and machine-learning based mapping. In: PLDI '09

40. Wang Z, O'Boyle M (2018) Machine learning in compiler optimization. Proc IEEE

41. Wang Z, O'Boyle MF (2009) Mapping parallelism to multi-cores: A machine learning based approach. In: PPoPP '09

42. Wang Z, O'Boyle MF (2010) Partitioning streaming parallelism for multicores: a machine learning based approach. In: PACT '10

43. Wang Z, O'boyle MF (2013) Using machine learning to partition streaming programs. ACM TACO

44. Wang Z, et al. (2014) Automatic and portable mapping of data parallel programs to opencl for gpu-based heterogeneous systems. ACM TACO

45. Wang Z, et al. (2014) Exploitation of gpus for the parallelisation of probably parallel legacy code. In: CC '14

46. Wang Z, et al. (2014) Integrating profile-driven parallelism detection and machine-learning-based mapping. ACM TACO

47. Williams S, Oliker L, Vuduc RW, Shalf J, Yelick KA, Demmel J (2007) Optimization of sparse matrix-vector multiplication on emerging multicore platforms. In: SC

48. Williams S, Oliker L, Vuduc RW, Shalf J, Yelick KA, Demmel J (2009) Optimization of sparse matrix-vector multiplication on emerging multicore platforms. Parallel Computing

49. Yang X, Fang J, Chen J, Wu C, Tang T, Lu K (2017) High performance coordinate descent matrix factorization for recommender systems. In: $\mathrm{CF}$

50. Zhang C (2015) Mars: A 64-core armv8 processor. In: HotChips

51. Zhang P, et al. (2018) Auto-tuning streamed applications on intel xeon phi. In: IPDPS ' 18

52. Zhao Y, Li J, Liao C, Shen X (2018) Bridging the gap between deep learning and sparse matrix format selection. In: PPoPP 